\title{
Article \\ Fasting Normoglycemia after Intravenous Thrombolysis Predicts Favorable Long-Term Outcome in Non-Diabetic Patients with Acute Ischemic Stroke
}

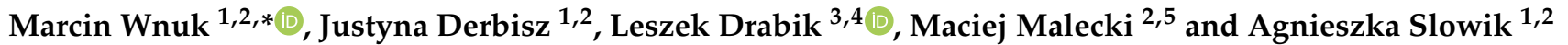 \\ 1 Department of Neurology, Jagiellonian University Medical College, 30-688 Krakow, Poland; \\ justyna.derbisz@gmail.com (J.D.); agnieszka.slowik@uj.edu.pl (A.S.) \\ 2 The University Hospital in Krakow, 30-688 Krakow, Poland; maciej.malecki@uj.edu.pl \\ 3 Department of Pharmacology, Jagiellonian University Medical College, 31-531 Krakow, Poland; \\ leszek.drabik@uj.edu.pl \\ 4 John Paul II Hospital, 31-202 Krakow, Poland \\ 5 Department of Metabolic Diseases, Jagiellonian University Medical College, 30-688 Krakow, Poland \\ * Correspondence: marcin.wnuk@uj.edu.pl
}

Citation: Wnuk, M.; Derbisz, J.; Drabik, L.; Malecki, M.; Slowik, A. Fasting Normoglycemia after Intravenous Thrombolysis Predicts Favorable Long-Term Outcome in Non-Diabetic Patients with Acute Ischemic Stroke. J. Clin. Med. 2021, 10, 3005. https://doi.org/10.3390/ jcm10143005

Academic Editors: Hyo Suk Nam, Byung Moon Kim and

Emmanuel Andrès

Received: 16 April 2021

Accepted: 28 June 2021

Published: 6 July 2021

Publisher's Note: MDPI stays neutral with regard to jurisdictional claims in published maps and institutional affiliations.

Copyright: (C) 2021 by the authors. Licensee MDPI, Basel, Switzerland. This article is an open access article distributed under the terms and conditions of the Creative Commons Attribution (CC BY) license (https:// creativecommons.org/licenses/by/ $4.0 /)$.

\begin{abstract}
Background: Only a few studies evaluated the role of fasting glucose levels after intravenous thrombolysis (IVT) in patients with acute ischemic stroke (AIS). Importantly, formal analysis concerning the prognostic role of fasting glucose levels in these patients with and without diabetes mellitus (DM) was not performed. Therefore, we assessed whether fasting normoglycemia (FNG) next morning after AIS treated with IVT was associated with 90-day functional outcome in diabetic and non-diabetic patients. Methods: We retrospectively analyzed 362 AIS patients treated with IVT at The University Hospital in Krakow. FNG was defined as glucose below $5.5 \mathrm{mmol} / \mathrm{L}$. A favorable outcome was defined as modified Rankin score (mRS) of 0-2 at day 90 after AIS onset. Results: At 3-month follow-up, FNG was associated with favorable outcome $(87.5 \%$ vs. $60.8 \%, p<0.001)$ and decreased risk of death ( $3.1 \%$ vs. $18.1 \%, p=0.002)$. Independent predictors of a favorable outcome for the whole group were: younger age (HR 0.92, 95\%CI 0.89-0.95), lower NIHSS score after IVT (HR 0.70, 95\%CI 0.65-0.76), lower maximal systolic blood pressure within $24 \mathrm{~h}$ after IVT (HR 0.92, 95\%CI 0.89-0.95) and FNG (HR 4.12, 95\%CI 1.38-12.35). Association between FNG and mortality was found in univariable (HR 1.47, 95\%CI 0.04-0.62) but not in multivariable analysis (HR 0.23, 95\%CI 0.03-1.81). In subgroup analyses, FNG was an independent predictor of favorable outcome (HR $5.96,95 \%$ CI 1.42-25.1) only in patients without DM. Conclusions: FNG next morning after IVT is an independent protective factor for a favorable long-term outcome in non-diabetic AIS patients.
\end{abstract}

Keywords: stroke; thrombolysis; fasting hyperglycemia; fasting normoglycemia; long-term outcome

\section{Introduction}

The prognostic significance of admission glucose levels in patients with acute ischemic stroke (AIS) treated with intravenous thrombolysis (IVT) is well established [1]. Hyperglycemia on admission has been associated with worse functional outcomes and increased mortality within 3 months after IVT in patients with or without diabetes mellitus (DM) [2].

However, only a few studies so far have evaluated the role of fasting glucose levels after IVT in patients with AIS [3,4]. Fasting hyperglycemia the next day or 2-5 days after IVT was associated with worse 3-month functional outcomes and increased mortality [3,4]. Importantly, the association with outcome was stronger for fasting glucose levels than for admission ones [3]. Diabetes mellitus was not found to be correlated with a 90-day poor clinical outcome as assessed with modified Rankin scale (mRS); however, the formal analysis concerning the prognostic role of fasting glucose levels in AIS patients treated with IVT according to the presence of DM was not performed [3,4]. To the best of our knowledge, no 
studies so far evaluated the impact of fasting glucose next morning after IVT in diabetic and non-diabetic patients on a 90-day clinical outcome.

The recent Stroke Hyperglycemia Insulin Network Effort (SHINE) trial, performed in AIS patients with concomitant hyperglycemia, evaluated the impact of intensive versus standard insulin therapy during the first $72 \mathrm{~h}$ from symptom onset on a 90-day functional outcome [5]. Although the analysis, adjusted for IVT or mechanical thrombectomy (MT) use, did not show any significant difference in long-term prognosis, it occurred that DM was present in around $80 \%$ of patients in both treated subgroups, and, consequently, the results may not be generalizable for a whole stroke population, including patients without pre-existent DM [5].

Therefore, the aim of the present study was to search whether fasting normoglycemia (FNG) the next day after IVT was associated with long-term outcomes in a large cohort of AIS patients according to the presence of DM.

\section{Materials and Methods}

The data supporting the results of this study are available from the corresponding author upon reasonable request from any qualified investigator.

\subsection{Patients}

The study was designed as a retrospective analysis of the prospectively collected data of 1209 AIS patients from the Krakow Stroke Data Bank, the registry conducted in the single stroke center, The University Hospital in Krakow, from the year 2007. Finally, the study included 362 AIS patients (29.9\%), all of Caucasian origin, treated with IVT between June 2014 and December 2018. We collected the data on demographics, the presence of vascular risk factors, etiology of AIS and National Institutes of Health Stroke Scale (NIHSS) on admission and after IVT. The diagnosis of DM was made as described previously [6]. In brief, patients were diagnosed with DM based on the previous medical history or the use of insulin or antidiabetic oral drugs before the onset of stroke [6].

The outcome was measured with mRS at day 90 from AIS onset, and a favorable outcome was defined as an mRS score of $0-2$, similarly to the previous studies investigating the prognostic role of fasting hyperglycemia [3,4]. Additionally, an excellent outcome was defined as an mRS score of 0-1 at day 90 after AIS onset. We also evaluated 3-month mortality. Bleeding brain complications due to IVT were defined in accordance with the ECASS-1 classification [7]. As higher systolic blood pressure (SBP) was found to increase the risk of symptomatic intracranial hemorrhage in the previous stroke registries [8,9], we additionally noted the maximal SBP value within $24 \mathrm{~h}$ after IVT.

\subsection{Glucose Measurements}

We evaluated serum glucose levels in each patient the next morning after IVT and overnight fasting. Fasting normoglycemia and hyperglycemia were defined as the glucose levels below $5.5 \mathrm{mmol} / \mathrm{L}(100 \mathrm{mg} \%$ ) and equal or above this value, respectively, in accordance with the American Diabetes Association's Standards of Care [10]. Patients with hyperglycemia greater than $10 \mathrm{mmol} / \mathrm{L}$ were treated with four subcutaneous insulin injections daily with doses adjusted to the current level of glucose [11].

The study was approved by the Jagiellonian University Ethical Committee (KBET 54/B/2007). All patients gave informed consent to participate in the study, which was either written or verbal in the presence of at least two physicians in case of inability to use the dominant hand because of AIS.

\subsection{Statistics}

The continuous variables were presented as mean and standard deviation (SD), and in the case of categorical data, counts and percentages were shown. Continuous variables were tested for normality with the use of the Shapiro-Wilk test and then compared, as appropriate, by a Student's t-test or by the Mann-Whitney U test. The multivariable logistic 
regression model comprised only those variables that showed a $p$-value of $<0.1$ in the univariable analysis. We considered a $p$-value of 0.05 (two-sided) as statistically significant and performed all statistical analyses with the use of STATISTICA version 13 (Statsoft Inc, Tulsa, OK, USA).

\section{Results}

\subsection{Patient Characteristics}

The characteristics of 362 AIS patients treated with IVT were summarized in Table 1. Among patients, 108 (29.8\%) underwent additionally MT.

Patients with FNG were younger, had lower Body Mass Index (BMI), lower NIHSS score before and after treatment with IVT and lower maximal SBP within $24 \mathrm{~h}$ after IVT in comparison to those with fasting hyperglycemia (Table 1).

\subsection{Association between FNG and Favorable Outcome}

\subsubsection{All Patients}

A favorable outcome applied to 231 (63.8\%) patients at 3-month follow-up. Patients with favorable outcome were younger (median, interquartile range IQR 70 (59-79) vs. 78 (69-83) years, $p<0.001)$, less often women $(43.7 \%$ vs. $59.5 \%, p=0.005)$, less often suffered from hypertension (77.5\% vs. $95.0 \%, p<0.001)$, had lower fasting glucose levels (median, IQR 6.4 (5.5-7.9) vs. 6.9 (6.1-8.6) $\mathrm{mmol} / \mathrm{L}, p=0.004)$, lower NIHSS score on admission (mean \pm standard deviation (SD), $9.7 \pm 6.0$ vs. $16.3 \pm 6.0, p<0.001$ ) and after IVT (mean \pm SD, $4.5 \pm 4.3$ vs. $16.0 \pm 7.6, p<0.001$ ), lower value of maximal SBP within $24 \mathrm{~h}$ after IVT (145 (126-160) vs. $146(137-165) \mathrm{mmHg}, p=0.048)$ and less often experienced bleeding brain complications $(10.0 \%$ vs. $41.3 \%, p<0.001)$ compared with the remainder (Supplemental Table S1, for subgroup of patients treated only with IVT and without MT see also Supplemental Table S2).

Patients with FNG had a higher prevalence of a favorable 3-month outcome than those with fasting hyperglycemia (Table 1, Figure 1). The independent predictors of favorable long-term outcome for the whole group were: younger age, lower NIHSS score after IVT, lower maximal SBP and FNG (Table 2). In the subgroup of patients treated only with IVT (without MT), favorable long-term outcome was predicted by age and NIHSS score after IVT. The association between FNG and outcome for these patients was found in the univariable analysis (Supplemental Figure S1, Supplemental Table S3). The optimal cutoff value of glucose for predicting $\mathrm{mRS} 0-2$ was $5.49 \mathrm{mmol} / \mathrm{L}$. Sensitivity and specificity using this cutoff value were $86.7 \%$ and $59.2 \%$, respectively. 
Table 1. Baseline characteristics of patients according to glycemia $<5.5 \mathrm{mmol} / \mathrm{L}$ and diabetes mellitus (DM).

\begin{tabular}{|c|c|c|c|c|c|c|c|c|c|}
\hline & \multicolumn{3}{|c|}{ Whole Group } & \multicolumn{3}{|c|}{ With DM } & \multicolumn{3}{|c|}{ Without DM } \\
\hline & $\begin{array}{c}\text { Glucose }<5.5 \\
\mathrm{mmol} / \mathrm{L} \\
n=69\end{array}$ & $\begin{array}{c}\text { Glucose } \geq 5.5 \\
\mathrm{mmol} / \mathrm{L} \\
n=293\end{array}$ & $p$-Value & $\begin{array}{c}\text { Glucose }<5.5 \\
\mathrm{mmol} / \mathrm{L} \\
n=12\end{array}$ & $\begin{array}{c}\text { Glucose } \geq 5.5 \\
\mathrm{mmol} / \mathrm{L} \\
n=93\end{array}$ & $p$-Value & $\begin{array}{c}\text { Glucose }<5.5 \\
\mathrm{mmol} / \mathrm{L} \\
n=57\end{array}$ & $\begin{array}{c}\text { Glucose } \geq 5.5 \\
\mathrm{mmol} / \mathrm{L} \\
n=200\end{array}$ & $p$-Value \\
\hline Age (years) & $70(54-78)$ & $73(64-82)$ & 0.005 & $78(71-80)$ & $74(65-82)$ & 0.331 & $65(53-75)$ & $72(64-82)$ & $<0.001$ \\
\hline Women, $n(\%)$ & $30(43.5)$ & $147(50.2)$ & 0.317 & $4(33.3)$ & $47(50.5)$ & 0.360 & $26(45.6)$ & $100(50.0)$ & 0.559 \\
\hline BMI $\left(\mathrm{kg} / \mathrm{m}^{2}\right)$ & $25.8(23.4-27.8)$ & $27.1(24.4-30.1)$ & 0.013 & $24.9(23.4-27.0)$ & $29.1(26.1-31.8)$ & 0.001 & $25.9(23.4-28.7)$ & $25.9(23.9-29.4)$ & 0.578 \\
\hline Hypertension, $n(\%)$ & $54(78.3)$ & $250(85.3)$ & 0.150 & $11(91.7)$ & $87(93.5)$ & 0.584 & $43(75.4)$ & $163(81.5)$ & 0.311 \\
\hline Ischemic heart disease, $n(\%)$ & $14(20.3)$ & $70(23.9)$ & 0.523 & $3(25.0)$ & $30(32.3)$ & 0.749 & $11(19.3)$ & $40(20.0)$ & 0.907 \\
\hline Atrial fibrillation, $n(\%)$ & $16(23.2)$ & $90(30.7)$ & 0.216 & $3(25.0)$ & $36(38.7)$ & 0.528 & $13(22.8)$ & $54(27.0)$ & 0.525 \\
\hline Hypercholesterolemia, $n(\%)$ & $29(42.0)$ & $88(30.0)$ & 0.055 & $7(58.3)$ & $30(32.3)$ & 0.108 & $22(38.6)$ & $58(29.0)$ & 0.167 \\
\hline Smoking, $n(\%)$ & $12(17.7)$ & $43(15.2)$ & 0.736 & $1(8.3)$ & $10(10.8)$ & 1.00 & $11(19.3)$ & $33(17.2)$ & 0.812 \\
\hline Previous stroke, $n(\%)$ & $12(17.4)$ & $54(18.40$ & 0.840 & $2(16.7)$ & $21(22.6)$ & 1.00 & $10(17.5)$ & $33(16.5)$ & 0.852 \\
\hline \multicolumn{10}{|l|}{ Stroke etiology, $n(\%)$} \\
\hline $\begin{array}{ll}- & \text { large-vessel disease } \\
- & \text { small-vessel disease } \\
- & \text { cardioembolic } \\
- & \text { other } \\
- & \text { undetermined }\end{array}$ & $\begin{aligned} 10 & (14.5) \\
2 & (2.9) \\
19 & (27.5) \\
5 & (7.3) \\
33 & (47.8)\end{aligned}$ & $\begin{aligned} 39 & (13.3) \\
1 & (0.3) \\
100 & (34.1) \\
12 & (4.1) \\
141 & (48.1)\end{aligned}$ & 0.172 & $\begin{array}{c}4(33.3) \\
0(0.0) \\
3(25.0) \\
0(0.0) \\
5(41.7)\end{array}$ & $\begin{aligned} 14 & (15.0) \\
1 & (1.1) \\
38 & (40.9) \\
2 & (2.1) \\
38 & (40.9)\end{aligned}$ & 0.534 & $\begin{aligned} 6 & (10.5) \\
2 & (3.5) \\
16 & (28.1) \\
5 & (8.8) \\
28 & (49.1)\end{aligned}$ & $\begin{array}{c}25(12.5) \\
0(0.0) \\
62(31.0) \\
10(5.0) \\
103(51.5)\end{array}$ & 0.077 \\
\hline Mechanical thrombectomy, $n(\%)$ & $26(37.7)$ & $82(28.0)$ & 0.113 & $1(8.3)$ & $33(35.5)$ & 0.978 & $25(43.9)$ & $49(24.5)$ & 0.004 \\
\hline $\begin{array}{l}\text { Time from stroke onset to } \\
\text { thrombolysis (min) }\end{array}$ & $138(99-176)$ & 135 (95-183) & 0.655 & $115(91-156)$ & $135(96-185)$ & 0.243 & $140(100-190)$ & $135(94-180)$ & 0.998 \\
\hline NIHSS score on admission & $10.3 \pm 6.6$ & $12.3 \pm 6.8$ & 0.026 & $8.7 \pm 4.9$ & $12.3 \pm 6.4$ & 0.060 & $10.7 \pm 6.1$ & $12.3 \pm 7.0$ & 0.122 \\
\hline NIHSS score after r-tPA & $6.3 \pm 6.6$ & $9.0 \pm 7.9$ & 0.012 & $7.0 \pm 5.1$ & $8.4 \pm 7.0$ & 0.665 & $6.1 \pm 6.9$ & $9.3 \pm 8.3$ & 0.010 \\
\hline \multicolumn{10}{|l|}{$\begin{array}{l}\text { Post-MT hemorrhagic brain } \\
\text { complications, } n(\%)\end{array}$} \\
\hline $\begin{array}{ll}- & \text { no complication } \\
- & \text { HI type } 1 \\
- & \text { HI type 2 } \\
- & \text { PH type 1 } \\
- & \text { PH type 2 }\end{array}$ & $\begin{aligned} 58 & (84.1) \\
4 & (5.8) \\
4 & (5.8) \\
2 & (2.9) \\
1 & (1.5)\end{aligned}$ & $\begin{array}{c}229(78.2) \\
21(7.2) \\
20(6.8) \\
12(4.1) \\
11(3.8)\end{array}$ & 0.810 & $\begin{array}{c}11(91.7) \\
1(8.3) \\
0(0.0) \\
0(0.0) \\
0(0.0)\end{array}$ & $\begin{array}{c}73(78.5) \\
6(6.4) \\
7(7.5) \\
4(4.3) \\
3(3.2)\end{array}$ & 0.718 & $\begin{array}{l}47(82.5) \\
3(5.3) \\
4(7.0) \\
2(3.5) \\
1(1.7)\end{array}$ & $\begin{array}{c}156(78.0) \\
15(7.5) \\
13(6.5) \\
8(4.0) \\
8(4.0)\end{array}$ & 0.892 \\
\hline
\end{tabular}


Table 1. Cont.

\begin{tabular}{|c|c|c|c|c|c|c|c|c|c|}
\hline & \multicolumn{3}{|c|}{ Whole Group } & \multicolumn{3}{|c|}{ With DM } & \multicolumn{3}{|c|}{ Without DM } \\
\hline & $\begin{array}{c}\text { Glucose }<5.5 \\
\mathrm{mmol} / \mathrm{L} \\
n=69\end{array}$ & $\begin{array}{c}\text { Glucose } \geq 5.5 \\
\mathrm{mmol} / \mathrm{L} \\
n=293\end{array}$ & $p$-Value & $\begin{array}{c}\text { Glucose }<5.5 \\
\mathrm{mmol} / \mathrm{L} \\
n=12\end{array}$ & $\begin{array}{c}\text { Glucose } \geq 5.5 \\
\mathrm{mmol} / \mathrm{L} \\
n=93\end{array}$ & $p$-Value & $\begin{array}{c}\text { Glucose }<5.5 \\
\mathrm{mmol} / \mathrm{L} \\
n=57\end{array}$ & $\begin{array}{c}\text { Glucose } \geq 5.5 \\
\mathrm{mmol} / \mathrm{L} \\
n=200\end{array}$ & $p$-Value \\
\hline $\begin{array}{l}\text { Maximal SBP within } 24 \mathrm{~h} \text { after } \\
\text { r-tPA (mmHg) }\end{array}$ & $140(120-150)$ & $147(135-164)$ & 0.002 & $140(125-150)$ & $148(135-160)$ & 0.285 & $140(120-150)$ & $146(134-165)$ & 0.005 \\
\hline $\begin{array}{l}\text { Maximal DBP within } 24 \mathrm{~h} \text { after } \\
\text { r-tPA (mmHg) }\end{array}$ & $80(71-83)$ & $80(70-90)$ & 0.101 & $80(70-80)$ & $80(70-90)$ & 0.459 & $80(72-85)$ & $80(71-90)$ & 0.124 \\
\hline Fasting glucose (mmol/L) & $5.0(4.7-5.3)$ & $6.9(6.2-8.6)$ & $<0.001$ & $4.4(3.6-4.7)$ & $8.1(6.5-11.5)$ & $<0.001$ & $5.2(4.9-5.3)$ & $6.7(6.1-7.8)$ & $<0.001$ \\
\hline Creatinine $(\mu \mathrm{mol} / \mathrm{L})$ & $78(69-98)$ & $82(68-97)$ & 0.572 & $103(72-122)$ & $83(64-99)$ & 0.177 & $74(67-95)$ & $82(69-92)$ & 0.202 \\
\hline mRS $0-1,90$ days, $n(\%)$ & $53(82.8)$ & $153(53.1)$ & $<0.001$ & $7(63.6)$ & $42(45.6)$ & 0.259 & $46(86.8)$ & $111(56.6)$ & $<0.001$ \\
\hline mRS $0-2,90$ days, $n(\%)$ & $56(87.5)$ & $175(60.8)$ & $<0.001$ & $8(72.7)$ & $54(58.7)$ & 0.368 & $48(90.6)$ & $121(61.7)$ & $<0.001$ \\
\hline Death $(\mathrm{mRS}=6), 90$ days, $n(\%)$ & $2(3.1)$ & $52(18.1)$ & 0.002 & $1(9.1)$ & 15 (16.3) & 1.000 & $1(1.9)$ & 37 (18.9) & 0.001 \\
\hline
\end{tabular}

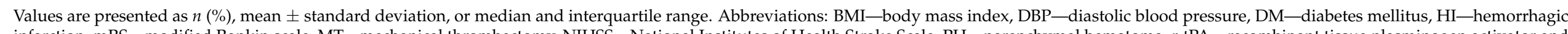

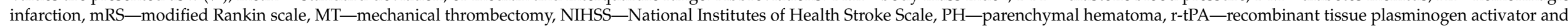
SBP—systolic blood pressure. 


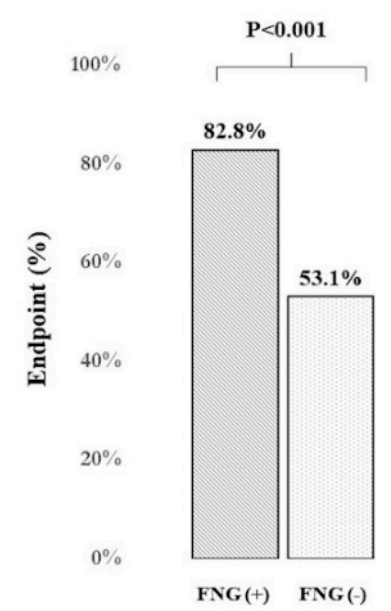

A
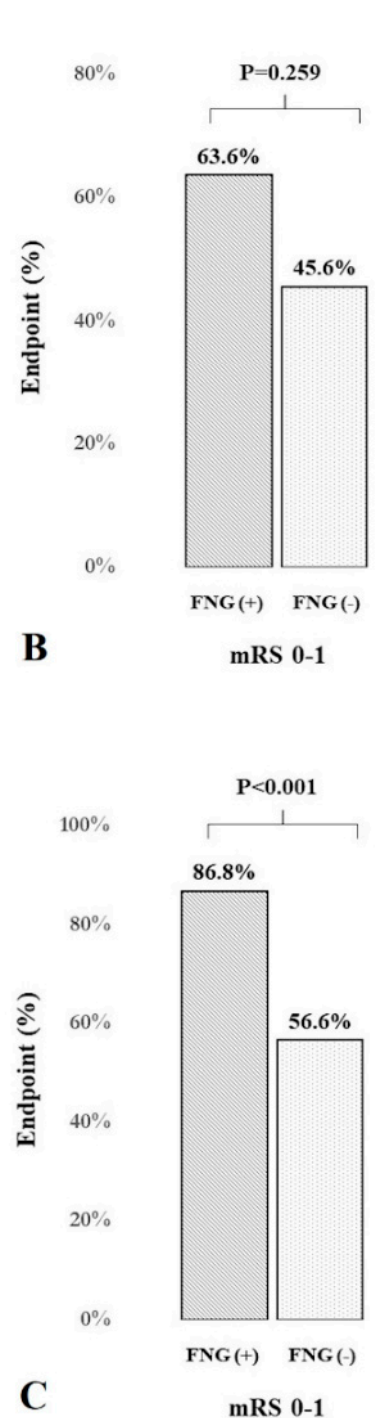

$\mathrm{P}<0.001$

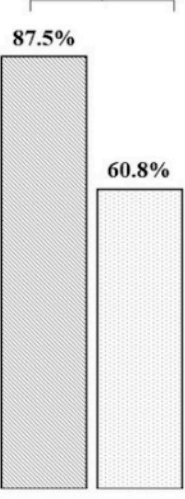

FNG (+) FNG (-)

mRS 0-2

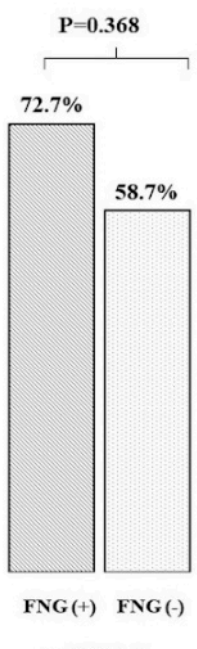

mRS 0-2

$\mathrm{P}<0.001$

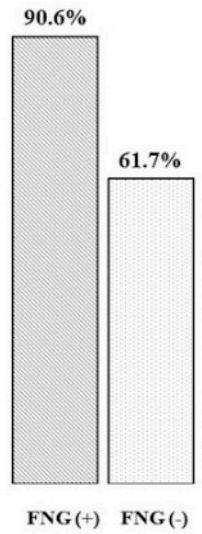

mRS 0-2

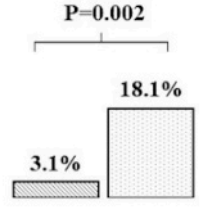

FNG (+) FNG (-)

Death

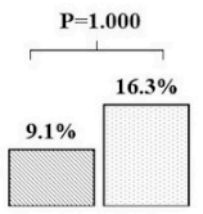

FNG (+) FNG (-)

Death

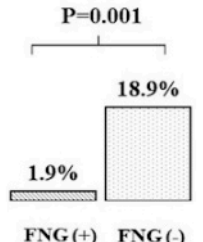

Death

Figure 1. The proportion of patients with a favorable (mRS 0-2) or excellent (mRS 0-1) outcome and those who died ( $\mathrm{mRS}=6$ ) according to the presence of fasting normoglycemia in the whole group (A) and patients with (B) and without diabetes mellitus (C). 
Table 2. The multivariable logistic regression model for a favorable (mRS 0-2) and excellent (mRS 0-1) 3-month clinical outcome.

\begin{tabular}{|c|c|c|c|c|c|c|}
\hline \multicolumn{7}{|c|}{ Favorable Outcome (mRS 0-2) } \\
\hline & \multicolumn{3}{|c|}{ Univariable } & \multicolumn{3}{|c|}{ Multivariable } \\
\hline $\begin{array}{l}\text { 90-day favorable clinical outcome, } \\
\text { diabetic + non-diabetic patients }\end{array}$ & HR & $95 \% \mathrm{CI}$ & $p$-Value & HR & $95 \%$ CI & $p$-Value \\
\hline Age (per 1 year) & 0.95 & $0.94-0.97$ & $<0.001$ & 0.92 & $0.89-0.95$ & $<0.001$ \\
\hline Sex (female) & 0.53 & $0.34-0.83$ & 0.005 & - & - & - \\
\hline BMI (per 1 unit) & 0.96 & $0.92-1.02$ & 0.163 & - & - & - \\
\hline Atrial fibrillation & 0.57 & $0.35-0.91$ & 0.019 & - & - & \\
\hline NIHSS score after r-tPA (per 1 point) & 0.73 & $0.69-0.78$ & $<0.001$ & 0.70 & $0.65-0.76$ & $<0.001$ \\
\hline $\begin{array}{l}\text { Maximal SBP within } 24 \mathrm{~h} \text { after r-tPA (per } \\
1 \mathrm{mmHg} \text { ) }\end{array}$ & 0.99 & $0.98-0.99$ & 0.053 & 0.92 & $0.89-0.95$ & 0.037 \\
\hline Mechanical thrombectomy & 0.67 & $0.42-1.07$ & 0.091 & - & - & - \\
\hline $\begin{array}{l}\text { Hemorrhagic brain complications } \\
\text { (ECASS 1-3) }\end{array}$ & 0.16 & $0.09-0.28$ & $<0.001$ & - & - & - \\
\hline Fasting glucose $<5.5 \mathrm{mmol} / \mathrm{L}$ & 4.52 & $2.08-9.83$ & $<0.001$ & 4.12 & $1.38-12.35$ & 0.011 \\
\hline \multicolumn{7}{|c|}{ 90-day favorable clinical outcome, diabetic patients } \\
\hline Age (per 1 year) & 0.96 & $0.92-0.99$ & 0.352 & 0.87 & $0.80-0.95$ & 0.002 \\
\hline Sex (female) & 0.92 & $0.42-2.03$ & 0.841 & - & - & - \\
\hline BMI (per 1 unit) & 0.89 & $0.81-0.98$ & 0.018 & - & - & - \\
\hline Atrial fibrillation & 0.48 & $0.21-1.08$ & 0.075 & - & - & - \\
\hline Previous stroke & 0.41 & $0.16-1.06$ & 0.067 & - & - & - \\
\hline NIHSS score after r-tPA (per 1 point) & 0.77 & $0.70-0.85$ & $<0.001$ & 0.64 & $0.54-0.77$ & $<0.001$ \\
\hline $\begin{array}{l}\text { Hemorrhagic brain complications } \\
\text { (ECASS 1-3) }\end{array}$ & 0.25 & $0.09-0.68$ & 0.007 & - & - & - \\
\hline Creatinine (per $1 \mu \mathrm{mol} / \mathrm{L}$ ) & 0.98 & $0.97-0.99$ & 0.019 & 0.97 & $0.94-0.99$ & 0.018 \\
\hline Fasting glucose $<5.5 \mathrm{mmol} / \mathrm{L}$ & 1.88 & $0.47-1.54$ & 0.375 & - & - & - \\
\hline \multicolumn{7}{|c|}{ 90-day favorable clinical outcome, non-diabetic patients } \\
\hline Age (per 1 year) & 0.95 & $0.93-0.98$ & $<0.001$ & 0.94 & $0.90-0.98$ & 0.01 \\
\hline Sex (female) & 0.40 & $0.23-0.69$ & 0.011 & - & - & - \\
\hline BMI (per 1 unit) & 1.02 & $0.95-1.09$ & 0.585 & - & - & - \\
\hline Hypertension & 0.18 & $0.07-0.47$ & $<0.001$ & - & - & - \\
\hline $\begin{array}{l}\text { Maximal SBP within } 24 \mathrm{~h} \text { after r-tPA (per } \\
1 \mathrm{mmHg} \text { ) }\end{array}$ & 0.99 & $0.98-0.99$ & 0.012 & 1.03 & $1.01-1.05$ & 0.007 \\
\hline NIHSS score after r-tPA (per 1 point) & 0.71 & $0.66-0.77$ & $<0.001$ & 0.68 & $0.62-0.75$ & $<0.001$ \\
\hline $\begin{array}{l}\text { Hemorrhagic brain complications } \\
\text { (ECASS 1-3) }\end{array}$ & 0.13 & $0.07-0.25$ & $<0.001$ & - & - & - \\
\hline Fasting glucose $<5.5 \mathrm{mmol} / \mathrm{L}$ & 5.95 & $2.27-15.6$ & $<0.001$ & 5.96 & $1.42-25.1$ & 0.015 \\
\hline
\end{tabular}


Table 2. Cont.

\begin{tabular}{|c|c|c|c|c|c|c|}
\hline \multicolumn{7}{|c|}{ Excellent Outcome (mRS 0-1) } \\
\hline & \multicolumn{3}{|c|}{ Univariable } & \multicolumn{3}{|c|}{ Multivariable } \\
\hline $\begin{array}{l}\text { 90-day excellent clinical outcome, } \\
\text { diabetic }+ \text { non-diabetic patients }\end{array}$ & HR & $95 \% \mathrm{CI}$ & $p$-Value & HR & $95 \%$ CI & $p$-Value \\
\hline Sex (female) & 0.59 & $0.38-0.90$ & 0.015 & - & - & - \\
\hline BMI (per 1 unit) & 0.96 & $0.96-1.01$ & 0.126 & - & - & - \\
\hline Atrial fibrillation & 0.63 & $0.40-0.99$ & 0.049 & - & - & - \\
\hline NIHSS score after r-tPA (per 1 point) & 0.73 & $0.69-0.77$ & $<0.001$ & 0.71 & $0.66-0.76$ & $<0.001$ \\
\hline $\begin{array}{l}\text { Maximal SBP within } 24 \mathrm{~h} \text { after r-tPA (per } \\
1 \mathrm{mmHg} \text { ) }\end{array}$ & 0.99 & $0.98-0.99$ & 0.014 & - & - & - \\
\hline Mechanical thrombectomy & 0.53 & $0.33-0.84$ & 0.007 & - & - & - \\
\hline $\begin{array}{l}\text { Hemorrhagic brain complications } \\
\text { (ECASS 1-3) }\end{array}$ & 0.17 & $0.10-0.30$ & $<0.001$ & - & - & - \\
\hline Fasting glucose $<5.5 \mathrm{mmol} / \mathrm{L}$ & 4.25 & $2.13-8.47$ & $<0.001$ & 3.47 & $1.32-9.14$ & 0.012 \\
\hline \multicolumn{7}{|c|}{ 90-day excellent clinical outcome, diabetic patients } \\
\hline Age (per 1 year) & 0.98 & $0.94-1.02$ & 0.226 & - & - & - \\
\hline Sex (female) & 1.52 & $0.70-3.32$ & 0.289 & - & - & - \\
\hline BMI (per 1 unit) & 0.89 & $0.81-0.98$ & 0.019 & - & - & - \\
\hline NIHSS score after r-tPA (per 1 point) & 0.70 & $0.62-0.80$ & $<0.001$ & 0.69 & $0.60-0.79$ & $<0.001$ \\
\hline Mechanical thrombectomy & 0.42 & $0.18-1.00$ & 0.050 & - & - & - \\
\hline $\begin{array}{l}\text { Hemorrhagic brain complications } \\
\text { (ECASS 1-3) }\end{array}$ & 0.27 & $0.09-0.81$ & 0.019 & - & - & - \\
\hline Creatinine (per $1 \mu \mathrm{mol} / \mathrm{L}$ ) & 0.99 & $0.97-0.99$ & 0.040 & 0.98 & $0.96-0.99$ & 0.021 \\
\hline Fasting glucose $<5.5 \mathrm{mmol} / \mathrm{L}$ & 2.08 & $0.57-7.61$ & 0.267 & - & - & - \\
\hline \multicolumn{7}{|c|}{ 90-day excellent clinical outcome, non-diabetic patients } \\
\hline Age (per 1 year) & 0.95 & $0.93-0.97$ & $<0.001$ & 0.96 & $0.93-0.99$ & 0.02 \\
\hline Sex (female) & 0.37 & $0.22-0.63$ & $<0.001$ & 0.34 & $0.14-0.87$ & 0.024 \\
\hline BMI (per 1 unit) & 1.03 & $0.96-1.10$ & 0.474 & - & - & - \\
\hline Hypertension & 0.21 & $0.09-0.49$ & $<0.001$ & - & - & - \\
\hline $\begin{array}{l}\text { Maximal SBP within } 24 \mathrm{~h} \text { after r-tPA (per } \\
1 \mathrm{mmHg} \text { ) }\end{array}$ & 0.98 & $0.97-0.99$ & $<0.001$ & - & - & - \\
\hline NIHSS score after r-tPA (per 1 point) & 0.72 & $0.67-0.78$ & $<0.001$ & 0.71 & $0.65-0.77$ & $<0.001$ \\
\hline Mechanical thrombectomy & 0.59 & $0.34-1.03$ & 0.065 & & & \\
\hline $\begin{array}{l}\text { Hemorrhagic brain complications } \\
\text { (ECASS 1-3) }\end{array}$ & 0.14 & $0.07-0.28$ & $<0.001$ & - & - & - \\
\hline Fasting glucose $<5.5 \mathrm{mmol} / \mathrm{L}$ & 5.03 & $2.16-11.7$ & $<0.001$ & 3.47 & $1.10-12.2$ & 0.035 \\
\hline
\end{tabular}




\subsubsection{Subgroup Analyses According to Diabetes}

Patients without DM who presented with FNG in comparison to those with fasting hyperglycemia were younger, had lower NIHSS score after IVT, lower fasting glucose levels, lower maximal SBP and more often underwent additionally MT (Table 1). In nondiabetic patients, variables that predicted a favorable 3-month outcome were: younger age, lower NIHSS score after IVT, lower maximal SBP and FNG (Table 2). In the subgroup of non-diabetic patients treated only with IVT, a favorable 3-month outcome was predicted by age, NIHSS score after IVT, maximal SBP and FNG (Supplemental Table S3).

Patients with DM and FNG in comparison to those with fasting hyperglycemia had a lower BMI and lower fasting glucose levels (Table 1). The presence of FNG was not an independent predictor of favorable long-term outcome in diabetic patients in contrast to a younger age, lower NIHSS score after IVT and lower creatinine concentration (Table 2). In the subgroup of diabetic patients treated only with IVT, a favorable long-term outcome was predicted by age, BMI and NIHSS score after IVT (Supplemental Table S3).

\subsection{Association between FNG and Excellent Outcome}

\subsubsection{All Patients}

Patients with FNG had a higher prevalence of an excellent 3-month outcome than those with fasting hyperglycemia (Table 1, Figure 1).

An excellent outcome occurred in 206 (56.9\%) patients. Patients with excellent outcome were younger (median, IQR, 69.5 (59-78) vs. 77 (68-83) years, $p<0.001$ ), less often women $(43.7 \%$ vs. $56.9 \%, p=0.015)$, less often suffered from hypertension $(76.2 \%$ vs. $93.8 \%$, $p<0.001)$ and atrial fibrillation $(25.2 \%$ vs. $34.9 \%, p=0.049)$, less often underwent MT ( $24.3 \%$ vs. $37.7 \%, p=0.007$ ), had lower NIHSS score on admission (mean \pm SD, $9.1 \pm 5.7$ vs. $16.0 \pm 6.0, p<0.001)$ and after IVT (mean \pm SD, $3.9 \pm 3.8$ vs. $14.8 \pm 7.6, p<0.001)$, a lower maximal SBP within $24 \mathrm{~h}$ after IVT (median, IQR 145 (126-159) vs. 146.5 (136-166) $\mathrm{mmHg}, p=0.019)$, lower fasting glucose levels (median, IQR 6.3 (5.5-7.7) vs. 7.0 (6.1-8.8) $\mathrm{mmol} / \mathrm{L}, p<0.001)$ and less often experienced bleeding brain complications $(9.2 \%$ vs. $37.0 \%$, $p<0.001)$ compared with the remainder (Supplemental Table S1). In the multivariable logistic regression model, the excellent outcome was predicted by lower NIHSS score after IVT and the presence of FNG (Table 2). In the subgroup of patients treated only with IVT and without MT, an excellent 3-month outcome was predicted by age, NIHSS score after IVT and FNG (Supplemental Table S3).

\subsubsection{Subgroup Analyses According to Diabetes}

For non-diabetic patients, independent predictors of excellent outcome were lower age, female sex, lower NIHSS score after IVT and FNG (Table 2). In the subgroup of non-diabetic patients treated only with IVT, an excellent 3-month outcome was predicted by age and NIHSS score after IVT (Supplemental Table S3).

An excellent outcome for diabetics was predicted by a lower NIHSS score after IVT and lower creatinine concentration but not by FNG (Table 2). In the subgroup of patients with diabetes treated with IVT and without MT, the independent predictors of excellent outcome were BMI, NIHSS score after IVT and creatinine concentration (Supplemental Table S3).

\subsection{Association between FNG and Mortality}

At a 3-month follow-up, 54 (14.9\%) patients died. Patients with FNG had a lower risk of death than those with fasting hyperglycemia (Table 1, Figure 1). The association between FNG and mortality was found in the univariable model for the whole group and the subgroup of patients treated only with IVT (Table 3, Supplemental Table S4). The factors that independently predicted the risk of death were older age, higher NIHSS score after IVT and hemorrhagic brain complications for the whole group, and age and NIHSS score after IVT in the subgroup of patients treated only with IVT and without MT (Table 3, Supplemental Table S4). 
Table 3. The multivariable logistic regression model for the risk of death.

\begin{tabular}{|c|c|c|c|c|c|c|}
\hline \multirow[b]{2}{*}{$\begin{array}{l}\text { 90-Day Risk of Death, Diabetic + } \\
\text { Non-Diabetic Patients }\end{array}$} & \multicolumn{3}{|c|}{ Univariable } & \multicolumn{3}{|c|}{ Multivariable } \\
\hline & HR & $95 \%$ CI & $p$-Value & HR & $95 \% \mathrm{CI}$ & $p$-Value \\
\hline Age (per 1 year) & 1.05 & $1.02-1.08$ & $<0.001$ & 1.07 & $1.03-1.11$ & $<0.001$ \\
\hline Sex (female) & 1.48 & $0.82-2.66$ & 0.189 & - & - & - \\
\hline BMI (per 1 unit) & 1.07 & $0.94-1.08$ & 0.870 & - & - & - \\
\hline NIHSS score after r-tPA (per 1 point) & 1.24 & $1.17-1.31$ & $<0.001$ & 1.22 & $1.15-1.29$ & $<0.001$ \\
\hline $\begin{array}{l}\text { Hemorrhagic brain complications } \\
\text { (ECASS 1-3) }\end{array}$ & 6.70 & $3.59-12.5$ & $<0.001$ & 2.66 & $1.19-5.91$ & 0.017 \\
\hline $\begin{array}{l}\text { Maximal SBP within } 24 \mathrm{~h} \text { after r-tPA (per } \\
1 \mathrm{mmHg} \text { ) }\end{array}$ & 1.01 & $1.01-1.03$ & 0.014 & - & - & - \\
\hline Hypertension & 3.85 & $1.16-12.78$ & 0.028 & - & - & - \\
\hline Atrial fibrillation & 2.02 & $1.11-3.67$ & 0.021 & - & - & - \\
\hline Platelets (per 105/ $\mu \mathrm{L}$ ) & 0.65 & $0.40-1.04$ & 0.071 & - & - & - \\
\hline Fasting glucose $<5.5 \mathrm{mmol} / \mathrm{L}$ & 1.47 & $0.04-0.62$ & 0.009 & 0.23 & $0.03-1.81$ & 0.164 \\
\hline \multicolumn{7}{|l|}{ 90-day risk of death, diabetic patients } \\
\hline Age (per 1 year) & 1.03 & $0.98-1.09$ & 0.254 & - & - & - \\
\hline Sex (female) & 1.12 & $0.39-3.26$ & 0.832 & - & - & - \\
\hline BMI (per 1 unit) & 1.11 & $1.00-1.25$ & 0.057 & - & - & - \\
\hline NIHSS score after r-tPA (per 1 point) & 1.24 & $1.12-1.38$ & $<0.001$ & 1.27 & $1.12-1.42$ & $<0.001$ \\
\hline $\begin{array}{l}\text { Hemorrhagic brain complications } \\
\text { (ECASS 1-3) }\end{array}$ & 4.06 & $1.30-12.7$ & 0.016 & - & - & - \\
\hline Atrial fibrillation & 3.70 & $1.22-11.2$ & 0.021 & 5.12 & $1.31-20.1$ & 0.019 \\
\hline Platelets count (per 105/ $\mu \mathrm{L}$ ) & 0.37 & $0.12-1.11$ & 0.075 & - & - & - \\
\hline Fasting glucose $<5.5 \mathrm{mmol} / \mathrm{L}$ & 0.51 & $0.06-4.31$ & 0.539 & - & - & - \\
\hline \multicolumn{7}{|l|}{ 90-day risk of death, non-diabetic patients } \\
\hline Age (per 1 year) & 1.06 & $1.02-1.09$ & $<0.001$ & 1.07 & $1.02-1.11$ & 0.005 \\
\hline Sex (female) & 1.67 & $0.83-3.38$ & 0.153 & - & - & - \\
\hline BMI (per 1 unit) & 0.94 & $0.85-1.03$ & 0.190 & - & - & - \\
\hline NIHSS score after r-tPA (per 1 point) & 1.24 & $1.16-1.33$ & $<0.001$ & 1.20 & $1.12-1.29$ & $<0.001$ \\
\hline $\begin{array}{l}\text { Hemorrhagic brain complications } \\
\text { (ECASS 1-3) }\end{array}$ & 8.29 & $3.92-17.6$ & $<0.001$ & 2.93 & $1.12-7.69$ & 0.029 \\
\hline $\begin{array}{l}\text { Maximal SBP within } 24 \mathrm{~h} \text { after r-tPA (per } \\
1 \mathrm{mmHg} \text { ) }\end{array}$ & 1.02 & $1.01-1.04$ & 0.001 & - & - & - \\
\hline Hypertension & 5.44 & $1.27-23.4$ & 0.023 & - & - & - \\
\hline Fasting glucose $<5.5 \mathrm{mmol} / \mathrm{L}$ & 0.08 & $0.01-0.62$ & 0.015 & 0.07 & $0.01-2.10$ & 0.125 \\
\hline
\end{tabular}




\section{Discussion}

Our study is the first to show that the association between FNG and long-term functional outcome after AIS treated with IVT is limited to the patients without pre-existent DM. This association was present even when the group of AIS patients was restricted to those who were treated only with IVT and without MT. Although this observation might be biased by a small sample size of patients with DM, our results were similar to the conclusions coming from our previous study performed on AIS patients who underwent MT [6]. Similarly, in the recent study of more than one thousand Chinese AIS patients, it was found that admission glucose levels independently predicted worse clinical outcomes after IVT only in patients without DM [12]. Non-diabetic patients seemed to be less adjusted to increased glucose levels than diabetics. This observation also came from a large cohort of more than 20,000 patients in whom fasting or random hyperglycemia increased the risk of transfer to an intensive care unit or in-hospital mortality only in non-diabetics [13]. Moreover, the pathophysiological mechanisms underlying the response to hyperglycemia in patients with and without DM might be different as glucose levels affected enlargement of infarct size in non-diabetic patients in contrast to diabetics [14]. Interestingly, in non-diabetic patients, female sex decreased the chance of a long-term excellent outcome. This observation stayed in line with previous research showing that after adjustment for age, women suffered from more severe AIS on admission and had a worse 3-month functional outcome [15].

Our study revealed that FNG after IVT resulted in a 3 or 4-fold increase in the chance of an excellent or favorable long-term outcome, respectively, at 3-month follow-up in AIS patients. Our results stayed in accordance with previous studies performed on smaller patient populations, which showed that fasting hyperglycemia after IVT increased the risk of worse outcome 3 months after AIS onset [3,4]. However, in our study, the effect size of fasting glucose levels was higher than previously reported. Data coming from a greater study that used a more complex parameter termed the stress hyperglycemia ratio but based on fasting glucose and glycated hemoglobin levels also indirectly supported the role of fasting hyper- or normoglycemia as prognostic factors in AIS patients treated with IVT [16]. In our study, fasting glucose levels influenced long-term outcomes, as did other well-known risk factors such as age and NIHSS score [17], both on admission and after IVT [17,18]. Interestingly, the lower maximal SBP within $24 \mathrm{~h}$ after IVT also independently affected the 3-month favorable outcome after AIS. Similarly, the Chinese study of 433 patients treated with IVT supported the prognostic role of lower SBP and revealed that maintaining its levels below $159.5 \mathrm{mmHg}$ increased the probability of a favorable 3-month outcome [19].

We found that FNG decreased the chance of death within 3 months after AIS in patients treated with IVT in univariable analysis. In the previous studies, there was either no analysis concerning the association with mortality performed [4] or, similar to our findings, fasting glucose levels did not independently predict the risk of death in the multivariable analysis [3]. One of the possible explanations of the lack of association between FNG and mortality may be a small number of patients who died at the 3-month follow-up. Other factors might also exhibit a more important risk for long-term mortality after IVT, such as age or bleeding brain complications rate, as was shown in the Pomeranian Stroke Register in Poland during the 3-year post-AIS observation period [20].

Patients with normoglycemia were younger, had lower BMI and less often suffered from DM, suggesting that insulin resistance might play a key role in mediating the risk of a long-term unfavorable outcome [21]. On the other hand, in the Japanese trial of 4655 AIS patients, it was found that the association of insulin resistance with outcome was also maintained in non-diabetic and non-obese patients [22]. Finally, neither BMI nor the presence of DM predicted outcome in the multivariable logistic regression model in our study for the whole group of AIS patients treated with IVT.

Our study has important limitations. First, the character of the study was retrospective. Moreover, we did not monitor for a change in glucose levels in the forthcoming days after IVT. We also did not gather the information on whether patients developed DM after hospitalization. Second, the subgroup analyses, especially according to the DM, may be 
biased by the small sample size. Third, the confounders, such as age, NIHSS score and SBP value, had a significant influence on the outcome of this study. Fourth, the study included only patients of Caucasian origin; therefore, its results may not be generalized to the patients of other ethnicities. Fifth, the results reported here may not reflect a causeeffect relationship.

\section{Conclusions}

In conclusion, although FNG is an infrequent finding in patients with AIS treated with IVT, it increases the chance of a favorable and even an excellent 3-month outcome in non-diabetics. It seems reasonable to undertake future studies to develop the prognostic scales in AIS patients treated with IVT with FNG as one of the important factors.

Supplementary Materials: The following are available online at https:/ / www.mdpi.com/article / 10.3390/jcm10143005/s1, Supplemental Table S1. Baseline characteristics of patients according to modified Rankin scale (mRS). Supplemental Table S2. Baseline characteristics of patients treated only with intravenous thrombolysis (without mechanical thrombectomy) according to modified Rankin scale (mRS). Supplemental Table S3. Multivariable logistic regression model for favorable (mRS 0-2) and excellent (mRS 0-1) 3-month clinical outcome in patients treated only with intravenous thrombolysis (without mechanical thrombectomy). Supplemental Table S4. Multivariable logistic regression model for the risk of death in patients treated only with intravenous thrombolysis (without mechanical thrombectomy). Supplemental Figure S1. Patients treated only with intravenous thrombolysis (without mechanical thrombectomy) with favorable (mRS 0-2) or excellent (mRS 0-1) outcome and those who died according to the presence of fasting normoglycemia in the whole group (A), in the subgroup with (B), and without diabetes mellitus (C).

Author Contributions: M.W. (conceptualization, draft writing and editing), J.D. (data curation), L.D. (formal analysis, methodology and draft editing), M.M. (supervision and draft review), A.S. (supervision and draft review). All authors have read and agreed to the published version of the manuscript.

Funding: This research received no external funding.

Institutional Review Board Statement: The study was approved by the Jagiellonian University Ethical Committee (KBET 54/B/2007) and conducted according to the guidelines of the Declaration of Helsinki.

Informed Consent Statement: All patients gave informed consent to participate in the study, which was either written or verbal in the presence of at least two physicians in case of inability to use the dominant hand because of acute ischemic stroke.

Data Availability Statement: The data supporting the results of this study are available from the corresponding author upon reasonable request from any qualified investigator.

Conflicts of Interest: The authors declare no conflict of interest.

\section{References}

1. Poppe, A.; Majumdar, S.; Jeerakathil, T.; Ghali, W.; Buchan, A.; Hill, M. Admission Hyperglycemia Predicts a Worse Outcome in Stroke Patients Treated with Intravenous Thromobolysis. Diabetes Care 2009, 32, 3-8. [CrossRef] [PubMed]

2. Tsivgoulis, G.; Katsanos, A.H.; Mavridis, D.; Lambadiari, V.; Roffe, C.; MacLeod, M.J.; Sevcik, P.; Cappellari, M.; Nevšímalová, M.; Toni, D.; et al. Association of baseline hyperglycemia with outcomes of patients with and without diabetes with acute ischemic stroke treated with intravenous thrombolysis: A propensity score-matched analysis from the SITS-ISTR registry. Diabetes 2019, 68, 1861-1869. [CrossRef] [PubMed]

3. Osei, E.; Fonville, S.; Zandbergen, A.A.M.; Koudstaal, P.J.; Dippel, D.W.J.; den Hertog, H.M. Impaired fasting glucose is associated with unfavorable outcome in ischemic stroke patients treated with intravenous alteplase. J. Neurol. 2018, 265, 1426-1431. [CrossRef]

4. Cao, W.; Ling, Y.; Wu, F.; Yang, L.; Cheng, X.; Dong, Q. Higher fasting glucose next day after intravenous thrombolysis is independently associated with poor outcome in acute ischemic stroke. J. Stroke Cerebrovasc. Dis. 2015, 24, 100-103. [CrossRef] [PubMed]

5. Johnston, K.C.; Bruno, A.; Pauls, Q.; Hall, C.E.; Barrett, K.M.; Barsan, W.; Fansler, A.; Van De Bruinhorst, K.; Janis, S.; DurkalskiMauldin, V.L. Intensive vs Standard Treatment of Hyperglycemia and Functional Outcome in Patients with Acute Ischemic Stroke: The SHINE Randomized Clinical Trial. JAMA J. Am. Med. Assoc. 2019, 322, 326-335. [CrossRef] 
6. Wnuk, M.; Popiela, T.; Drabik, L.; Brzegowy, P.; Lasocha, B.; Wloch-Kopec, D.; Pulyk, R.; Jagiella, J.; Wiacek, M.; Kaczorowski, R.; et al. Fasting Hyperglycemia and Long-term Outcome in Patients with Acute Ischemic Stroke Treated with Mechanical Thrombectomy. J. Stroke Cerebrovasc. Dis. 2020, 29, 104774. [CrossRef] [PubMed]

7. Trouillas, P.; Von Kummer, R. Classification and pathogenesis of cerebral hemorrhages after thrombolysis in ischemic stroke. Stroke 2006, 37, 556-561. [CrossRef]

8. Wahlgren, N.; Ahmed, N.; Eriksson, N.; Aichner, F.; Bluhmki, E.; Dávalos, A.; Erilä, T.; Ford, G.A.; Grond, M.; Hacke, W.; et al. Multivariable Analysis of Outcome Predictors and Adjustment of Main Outcome Results to Baseline Data Profile in Randomized Controlled Trials. Stroke 2008, 39, 3316-3322. [CrossRef]

9. Ahmed, N.; Wahlgren, N.; Brainin, M.; Castillo, J.; Ford, G.A.; Kaste, M.; Lees, K.R.; Toni, D. Relationship of Blood Pressure, Antihypertensive Therapy, and Outcome in Ischemic Stroke Treated With Intravenous Thrombolysis. Stroke 2009, 40, $2442-2449$. [CrossRef]

10. American Diabetes Association. Standards of medical care in diabetes 2014. Diabetes Care 2014, 37, S81-S90.

11. Powers, W.J.; Rabinstein, A.A.; Ackerson, T.; Adeoye, O.M.; Bambakidis, N.C.; Becker, K.; Biller, J.; Brown, M.; Demaerschalk, B.M.; Hoh, B.; et al. 2018 Guidelines for the Early Management of Patients With Acute Ischemic Stroke: A Guideline for Healthcare Professionals From the American Heart Association/American Stroke Association. Stroke 2018, 49, e46-e110. [CrossRef]

12. Fang, H.; Pan, Y.; Wang, Y.; Wang, C.; Wang, Y.; Zhong, L. Prognostic value of admission hyperglycemia on outcomes of thrombolysis in ischemic stroke patients with or without diabetes. Chin. Med. J. 2020, 133, 2244-2246. [CrossRef]

13. Tayek, C.J. Diabetes patients and non-diabetic patients intensive care unit and hospital mortality risks associated with sepsis. World J. Diabetes 2012, 3, 29. [CrossRef] [PubMed]

14. Shimoyama, T.; Kimura, K.; Uemura, J.; Saji, N.; Shibazaki, K. Elevated glucose level adversely affects infarct volume growth and neurological deterioration in non-diabetic stroke patients, but not diabetic stroke patients. Eur. J. Neurol. 2014, 21, 402-410. [CrossRef] [PubMed]

15. Fraticelli, L.; Freyssenge, J.; Claustre, C.; Buisson, M.; Bischoff, M.; Nighoghossian, N.; Derex, L.; El Khoury, C. Sex-Related Differences in Management and Outcome of Acute Ischemic Stroke in Eligible Patients to Thrombolysis. Cerebrovasc. Dis. 2019, 47, 196-204. [CrossRef]

16. Ngiam, J.N.; Cheong, C.W.S.; Leow, A.S.T.; Wei, Y.-T.; Thet, J.K.X.; Lee, I.Y.S.; Sia, C.-H.; Tan, B.Y.Q.; Khoo, C.-M.; Sharma, V.K.; et al. Stress Hyperglycaemia is Associated with Poor Functional Outcomes in Patients with Acute Ischaemic Stroke after Intravenous Thrombolysis. QJM Int. J. Med. 2020, 1-5. [CrossRef] [PubMed]

17. Bandettini di Poggio, M.; Finocchi, C.; Brizzo, F.; Altomonte, F.; Bovis, F.; Mavilio, N.; Serrati, C.; Malfatto, L.; Mancardi, G.L.; Balestrino, M. Management of acute ischemic stroke, thrombolysis rate, and predictors of clinical outcome. Neurol. Sci. 2019, 40, 319-326. [CrossRef]

18. Wu, Z.; Zeng, M.; Li, C.; Qiu, H.; Feng, H.; Xu, X.; Zhang, H.; Wu, J. Time-dependence of NIHSS in predicting functional outcome of patients with acute ischemic stroke treated with intravenous thrombolysis. Postgrad. Med. J. 2019, 95, 181-186. [CrossRef] [PubMed]

19. Wu, L.; Huang, X.; Wu, D.; Zhao, W.; Wu, C.; Che, R.; Zhang, Z.; Jiang, F.; Bian, T.; Yang, T.; et al. Relationship between Post-Thrombolysis Blood Pressure and Outcome in Acute Ischemic Stroke Patients Undergoing Thrombolysis Therapy. J. Stroke Cerebrovasc. Dis. 2017, 26, 2279-2286. [CrossRef]

20. Chwojnicki, K.; Kozera, G.; Sobolewski, P.; Fryze, W.; Nyka, W.M. Intravenous thrombolysis and three-year ischemic stroke mortality. Acta Neurol. Scand. 2017, 135, 540-545. [CrossRef] [PubMed]

21. Perova, N.V.; Ozerova, I.N.; Aleksandrovich, O.V.; Metel'skaia, V.A.; Shal'nova, S.A. Clinical value of insulin resistance in fasting normoglycemia. Kardiologiia 2011, 51, 49-54. [PubMed]

22. Ago, T.; Matsuo, R.; Hata, J.; Wakisaka, Y.; Kuroda, J.; Kitazono, T.; Kamouchi, M. Insulin resistance and clinical outcomes after acute ischemic stroke. Neurology 2018, 90, E1470-E1477. [CrossRef] [PubMed] 